\title{
Un análisis epistemológico del diagnóstico de depresión
}

Sandra Caponi ${ }^{1}$

CAPONI, S. An epistemological analysis of the diagnosis of depression. Interface Comunic., Saúde, Educ., v.13, n.29, p.327-38, abr./jun. 2009.

The definition of the diagnosis of depression is analyzed based on the reflections of authors such as Canguilhem, Hacking and Pignarre. The present study does not deny the existence of concrete situations that might correspond to such diagnosis, but rather aims at carrying out a strictly epistemological analysis, however incomplete, of the way in which different aspects of this classification are validated and legitimized. First, based on Hacking's theorizations, the way in which human classifications are built is analyzed. Next, the epistemological fragility of the descriptive diagnosis of depression in the DSM-IV is pointed out, based on Canguilhem's concepts of normality and pathology. Finally, based on Pignarre's studies, some difficulties inherent in etiological explanations for this pathology are analyzed. It is possible to conclude that the alarming frequency of this diagnosis is associated with the epistemological fragility of that classificatory category.

Keywords: Depression. Epistemology. Diagnostic classifications. Causality.
Tomando como punto de partida las reflexiones de autores como Canguilhem, Hacking y Pignarre, se analiza la definición del diagnóstico de depresión. No se pretende negar la existencia de situaciones concretas que puedan corresponder a ese diagnóstico, sino realizar un análisis estrictamente epistemológico, y necesariamente incompleto, del modo como los diferentes aspectos de esta clasificación se validan y legitiman. En primer lugar se analiza, a partir de las teorizaciones de Hacking, de que modo se construyen las clasificaciones humanas. En segundo lugar, a partir de los conceptos de normalidad y patología de Canguilhem, se destacan algunas fragilidades epistemológicas del diagnóstico descriptivo de depresión presentes en el DSM-IV. Por fin, a partir

fundamentalmente de los estudios de Pignarre, son analizadas algunas dificultades propias de las explicaciones etiológicas dadas a esa patología. Es posible concluir que la fragilidad epistemológica de esa categoría clasificatoria está directamente vinculada con la difusión de ese diagnóstico.

Palabras clave: Depresión. Epistemología. Clasificaciones diagnósticas. Causalidad.
${ }^{1}$ Graduada em Filosofia. Departamento de Saúde Pública, Universidade Federal de Santa

Catarina. Rua Esteves Junior, 605, ap 1414. Florianópolis, SC, Brasil. 88.015-130 sandracaponi@gmail.com 
Reiteradamente escuchamos hablar de una nueva epidemia que parece haberse diseminado por el mundo: la epidemia de depresión. Los gobiernos lanzan programas de divulgación como la reciente campaña iniciada en Francia por el Ministerio de Salud en noviembre de 2007 (Francia, 2007), los medios de comunicación divulgan los riesgos de esa temida enfermedad, los epidemiólogos anuncian el crecimiento alarmante de las tazas de depresión y presentan las más variadas correlaciones imaginables, los laboratorios están dedicados a buscar nuevos antidepresivos cada vez más sofisticados y con menos efectos colaterales.

Con el distanciamiento teórico que nos permite un análisis epistemológico, pretendo abordar los discursos y saberes referidos a la medicalización del sufrimiento, tomando como punto de partida la reflexión de autores como Canguilhem (1993); Hacking (2006a) y Pignarre (2001). Hoy, cuando la depresión parece haberse transformado en un diagnóstico recurrente a tal punto que ya es posible hablar de "epidemia", parece que debe ser problematizado el modo como son construidos esos nuevos diagnósticos referidos a sufrimientos cotidianos.

Para analizar la emergencia, consolidación y expansión del diagnóstico de depresión puede ser útil que recordemos la relación entre palabras y cosas tal como fue abordada por lan Hacking (Hacking, 2001). Este autor recuerda en su clase inaugural del College de France uno de los aforismos de La Gaia Ciencia de Nietzsche: "el nombre que damos a las cosas importa infinitamente más de lo que ellas son. Es suficiente crear nombres nuevos, apreciaciones nuevas, probabilidades nuevas para crear, más temprano o más tarde, cosas nuevas" (Nietzsche, 1981, p.67).

Aunque Nietzsche habla de cosas ese aforismo es aún más apropiado para hablar de personas, o más específicamente de "tipos de personas", de "clases de personas", de "categorías de personas".

Sabemos que el nombre que damos a los grupos humanos tiene, a diferencia del nombre que damos a las agrupaciones de cosas, efectos sobre los sujetos clasificados. Cuando clasificamos personas, cuando les atribuimos un diagnóstico, inevitablemente habrá consecuencias para esos sujetos, sean ellas previsibles o imprevisibles. Esto significa que, crear clasificaciones, definir "tipos de gente", implica necesariamente crear una relación interactiva.

Para Hacking entre el nombre y el objeto, entre la palabra y la cosa resulta necesario que exista una mediación. En el aforismo referido, Nietzsche no parece estar interesado en comprender esas mediaciones. Hacking, al contrario, prefiere afirmar que el nombre, la palabra, la clasificación, resultan insuficientes para explicar el modo como la ciencia modela los sujetos. Aunque una palabra pueda ofender o hasta herir a una determinada persona, eso no implica necesariamente que sea un modo de producir subjetividades. Para que eso ocurra, como Goffman (1988) ha sabido mostrarlo, se necesita mucho más que la simple enunciación.

\section{Diagnóstico y clasificación}

Para que un nombre "pueda iniciar su trabajo de creación" (Hacking, 2006a) resulta necesario, en primer lugar, que aquél que habla, aquél que enuncia una clasificación o un diagnóstico, aquél que nos sitúa en el interior de esa o de aquella categoría, esté imbuido de un cierto poder o reconocimiento. Será necesario que exista cierta autoridad conferida al sujeto de enunciación, por ejemplo, por determinadas instituciones. Esto significa afirmar que para que una clasificación tenga algún efecto sobre los sujetos clasificados deben existir por lo menos cinco variables en juego: (1) las personas situadas en el interior de la categoría $X$ (aquel que puede reconocerse como siendo depresivo, fóbico o TDAH) ; (2) la categoría X; (3) los expertos capaces de clasificar esos sujetos como siendo $X_{\text {; }}$ esos expertos deben estar situados en (4) instituciones, y edificar sus clasificaciones desde un cierto tipo de (5) conocimiento que permita diferenciar $X$ de no X (Hacking, 2005).

Aunque no es posible afirmar que existen dos modos idénticos de construir clases de sujetos, es posible identificar ciertas estrategias que las ciencias humanas, entre ellas la medicina, utilizaron a lo largo de su historia para crear categorías de personas. Hacking dio a ese conjunto de estrategias que articulan las instancias referidas (personas, clasificaciones, expertos, instituciones, conocimientos) el 
nombre de "motores de descubrimiento", que también pueden ser definidos como "motores para modelar personas".

Esos motores son: (1) Contar; (2) Cuantificar; (3) Crear normas; (4) establecer correlaciones; (5) medicalizar; (6) biologizar; (7) transformar en genético; (8) Normalizar; (9) burocratizar; (10) reivindicar la identidad. Dirá que: "Ios siete primeros motores de la lista sirven para identificar las personas; el octavo es un motor de práctica; el noveno es un motor de administración, el décimo es la resistencia de los sujetos a aquellos que poseen lo saber" (Hacking, 2005, p.14).

El motor que aparece en tercer lugar, crear normas, es, probablemente, el más relevante. Es a partir de esas normas que son construidas las estrategias de normalización y burocratización referidas en los motores octavo y noveno. Pero, el establecimiento de normas precisa de la cuantificación. Como Canguilhem evidenció en "Lo Normal y lo patológico", la medicina explica todas las enfermedades como desvíos con relación a una media estadística que indica la frecuencia de ese fenómeno en la población en general (Lecourt, 2006; Canguilhem, 1990). Para cada tipo de persona, para cada diagnóstico, para cada categoría surgirán diversos procesos de normalización. Aunque las estrategias de gestión y administración puedan ser diferentes su objetivo último parece ser el mismo: la restitución de la normalidad perdida.

\section{El diagnóstico de depresión}

Para que la depresión pueda transformarse en la epidemia del siglo, fue necesaria la creación de una red de pequeñas interacciones entre sujetos con frustraciones y sufrimientos cotidianos, y un aparato estadístico y médico cada vez más sofisticado de clasificación de síntomas, de localización cerebral, de diagnósticos por imagen.

No se trata de afirmar que la depresión es una mera invención histórica, una ficción socialmente creada con el objetivo de manipular a los sujetos. Parto de la certeza de que existe un fenómeno patológico que puede producir sufrimientos extremos. El problema que me interesa explorar es estrictamente epistemológico: de que modo es definido, validado y legitimado el diagnóstico de depresión. Creo que la fragilidad epistemológica de esa categoría clasificatoria puede ayudarnos a comprender su amplia difusión y su expansión a circunstancias que en otros contextos históricos y sociales jamás hubieran sido consideradas patológicas.

Retomando las variables identificadas por Hacking (2006a, 2005), podemos decir que para que el diagnóstico de depresión inicie su proceso de creación, para que pueda convertirse en una categoría clasificatoria deben existir: (1) las personas que puede reconocerse como perteneciendo a una categoría, esto es los sujetos que se reconocen y se identifican como "depresivos"; (2) la categoría, esto es la definición del diagnóstico y las múltiples variaciones posibles que se asocian con él: depresión con hiperactividad, depresión con ansiedad, depresión endógena, depresión exógena, leve, moderada, grave, etc. (3) los expertos capaces de clasificar esos sujetos como siendo depresivos (psiquiatras y médicos generales); esos expertos deben estar situados en (4) instituciones, sean ellas instituciones psiquiátricas o ambulatorio médico, y deben edificar sus clasificaciones desde un cierto tipo de (5) conocimiento que permita diferenciar X "depresión" de no X "no- depresión", hoy ese conocimiento se encuentra definido (para esos expertos) de manera objetiva en el CID 10 y en el DSM-IV (APA, 1994).

Recordemos, como son definidos por el DSM-IV los criterios para diagnosticar un episodio depresivo. Presencia de cinco (o más) de los nueve síntomas explicitados a seguir durante un período de duas semanas. Uno de los síntomas debe ser (1) poseer un estado de ánimo depresivo; (2) manifestar pérdida de interés o de la capacidad para el placer; (3) o pérdida o aumento del apetito; (4) insomnio o hipersomnia casi cada día; (5) agitación o enlentecimiento psicomotores; (6) fatiga o pérdida de energía; (7) sentimientos de inutilidad o de culpa excesivos o inapropiados; (8) disminución de la capacidad para pensar o concentrarse, o indecisión; (9) pensamientos recurrentes de muerte, ideas suicidas o tentativas de suicidio (APA, 1994).

En un episodio leve, deben estar presentes por lo menos dos de esos síntomas. El paciente sufre con la presencia de estos síntomas aunque es capaz de desempeñar la mayor parte de sus actividades 
cotidianas. En el caso de episodio moderado, están presentes cuatro o más síntomas y el paciente tiene dificultad para desempeñar actividades de rutina. El tratamiento considerado más eficaz es la asociación de psicofármacos, particularmente los llamados Inhibidores Específicos de Re-captación de Serotonina (ISRS), con psicoterapia.

Hasta aquí, los criterios para establecer un diagnóstico no se refieren a explicaciones etiológicas sino a la descripción y enumeración de síntomas. Aunque existan múltiples referencias a disfunciones cerebrales en la literatura, en la mayor parte de los casos se prescinde de estudios neurológicos por considerar que la frecuencia de aparición de los síntomas constituye una indicación suficiente para establecer un diagnóstico.

Entonces, surge una cuestión inevitable. Por que mecanismos ocurre ese desplazamiento que va del establecimiento de un diagnóstico descriptivo y no etiológico a la prescripción de una terapéutica basada en modificaciones químicas de funciones cerebrales? Este desplazamiento supone una explicación etiológica que, sin embargo, no interviene en los criterios para establecer el diagnóstico.

Para responder a esta cuestión deberemos detenernos en aquello que Hacking (2000) denominó los motores de descubrimiento, y analizar cuáles son las estrategias que posibilitan la articulación de esos motores en el caso específico de la depresión.

\section{Contar y establecer correlaciones}

Al analizar los números referidos a la depresión (Hacking, 2005), podemos observar que año tras año se multiplica el número de personas diagnosticadas. Según la OMS, esa enfermedad afecta actualmente aproximadamente 121 millones de personas y aunque las estimativas de prevalencia de depresión varían continuamente, las proyecciones para el año 2020 realizadas la sitúan en segundo lugar en el "ranking" de las enfermedades que más incapacidad producirán en el mundo (WHO, 2007).

En el caso de Brasil, datos divulgados por el "Portal da Saúde" (Brasil, 2007) del Ministerio de salud, indican que aproximadamente dez millones de brasileros sufren de depresión. En el año 2006, 41,5 mil individuos fueron internados debido a trastornos de humor, fundamentalmente depresión (Lobo, 2007). En Francia el site info-depression (Francia, 2007) notifica que son tres millones los diagnosticados en 2007. Datos de la OMS del año 2003, indican que

13-20\% de la población mundial presenta síntomas depresivos, siendo $2-3 \%$ de ese total atribuidos a individuos con trastornos afectivos graves, de lo cuales $15-30 \%$ cometen suicidio o son potenciales suicidas. Aún bajo tratamiento médico, y que $30 \%$ de los pacientes con diagnóstico depresivo no responden à terapia farmacológica. (Romeiro, 2003, p.354)

Los estudios demográficos sobre depresión revelan que las mujeres son más susceptibles que los hombres en una relación de 2:15, con edad media entre veinte y quarenta, aumentando el riego después de 65 años. En cuanto al estado civil, personas separadas o divorciadas presentan los mayores riesgos. Los hombres casados tienen menor disposición a la depresión que los solteros, ocurriendo lo opuesto entre mujeres casadas que presentan mayor riesgo con relación a las solteras (Romeiro, 2003). La OMS no considera significativa la diferencia de edad, sexo o clase social (WHO, 2007).

Por otra parte, y es posible establecer correlaciones (Hacking, 2005) entre la depresión y los diversos ciclos o etapas que caracterizan a la condición humana. Así, las estadísticas muestran que existe depresión en la infancia, aún en el feto, en la adolescencia, entre los veinte y cuarenta años, en la menopausia, después de los sessenta años. Estudios estadísticos indican el aumento de la depresión materna, post-parto, asociada a estrés en el trabajo, asociada a la vejez, asociada al uso de sustancias como alcohol o drogas, asociada al aislamiento social, al duelo (después de dos meses) (APA, 1994), a situaciones de frustración, a sentimientos de culpa, ansiedad, alimentación, sexualidad y sueño (Franco, 2005). Así, en cada momento de nuestra vida, hagamos lo que hagamos, estamos en riesgo de ser diagnosticados como deprimidos. 
Esa variedad prácticamente infinita de correlaciones posibles asociadas al diagnóstico de depresión, pone en evidencia cierta fragilidad epistemológica de esa categoría clasificatória. Pues, si toda y cualquier correlación es posible, deberemos concluir que estamos frente a una clasificación ambigua, con una capacidad predictiva ilimitada, donde nada parece escapar a su poder clasificatorio.

\section{Un diagnóstico descriptivo: entre normas y frecuencias}

Cuantificar y crear normas (Hacking, 2005, 2000) son, en el caso de la depresión, dos caras de una misma moneda. El Manual de Diagnóstico y Estadística de las Perturbaciones Mentales, el DSM-IV, publicado por la American Psychiatric Association en 1994, se transformó en el instrumento de referencia casi exclusivo para el área de salud mental y para los médicos generales (conjuntamente con el CID 10). Y por tratarse de un manual de diagnóstico y estadística, como su propio nombre lo indica, la definición de lo que debe considerarse normal o patológico está directamente asociada a la frecuencia de aparición de determinados comportamientos.

Las críticas que Canguilhem dirige a la asociación entre normalidad y frecuencia estadística, como estrategia para definir los valores normales y deseables de una función orgánica (Canguilhem, 1993) (nivel de colesterol, pulso cardíaco, presión arterial, etc.) resultan, en el caso del diagnóstico de depresión, aún más pertinentes y necesarias.

Recordemos que para Canguilhem el concepto de 'normal' es doble: de un lado nos remite a la noción de media estadística, constantes y tipos; de otro, se trata de un concepto valorativo que se refiere a aquello que es considerado como deseable en un determinado momento y en una determinada sociedad. El primer sentido es solidario al concepto bio-estadístico de salud enunciado por Boorse ( 1975). El segundo vincula lo normal con valores sociales o vitales: nos dice como una función o proceso "debería ser".

De hecho, aún si nos restringimos a los valores que deben ser considerados normales para un determinado órgano, veremos que "no encontraremos apenas un intervalo normal para cada función de un órgano: rigurosamente hablando, encontramos un número infinito de estos intervalos" (Nordenfelt, 1984, p.60). Veremos que existen infinitos ejemplos de variaciones y desvíos, de 'anomalías' que no pueden ser, en absoluto, consideradas de valor negativo o patológicas.

Canguilhem (1993, p.145) afirma que: "Lo normal, no tiene la rigidez de un determinante que vale para toda la especie, sino la flexibilidad de una norma que se transforma en relación a las condiciones individuales, entonces es claro que el límite entre el normal y el patológico es impreciso".

Una anomalía o un desvío de la frecuencia media no puede ser identificada inmediatamente a una patología. Las anomalías poseen valor neutro mientras las patologías poseen valor negativo. Así, una anomalía solo podrá ser considerada patológica si está vinculada a un sentimiento directo y concreto de sufrimiento, 'un sentimiento de vida contrariada'. No son las medias estadísticas, ni la fuga de los intervalos así llamados normales lo que indica el momento en el que se inicia una enfermedad, sino las dificultades que el organismo encuentra para dar respuestas a las demandas que su medio le impone.

Aquí aparece la primera fragilidad epistemológica del diagnóstico de depresión. El DSM-IV establece con que frecuencia la aparición de ciertas conductas y sentimientos debe ser considerada como indicador de una patología. Cuanto mayor sea el desvío de la frecuencia normal mayor será la patología.

EI DSM-IV se refiere a conductas y sentimientos de sujetos sin redes sociales, de comportamientos de individuos cuyos vínculos y relaciones amorosas, afectivas, laborales, en fin, cotidianas, pasan a estar en un segundo plano a la hora de definir el diagnóstico. El contexto preciso en el que surgen esos comportamientos serán o no considerados dependiendo de la competencia del médico, del tiempo disponible para la consulta, de la receptividad y escucha que el paciente encuentre en el profesional (Valentini, 2004).

Tomemos un caso extremo como es el duelo.

En el apartado (E) el DSM-IV afirma que los síntomas definidos en el apartado (A) "no se explican mejor por la presencia de duelo ( $p$. ej., después de la pérdida de un ser querido), si los síntomas persisten durante más de dos meses o se caracterizan por una acusada incapacidad funcional, 
preocupaciones mórbidas de inutilidad, ideación suicida, síntomas sicóticos o enlentecimiento psicomotor" (APA, 1994). Ante esta afirmación parece inevitable una pregunta: cuales son los nuevos modelos estadísticos que nos pueden llevar a concluir que una situación de duelo es "patológica" si excede los dos meses?

En ese caso, la atribución de un diagnóstico debería comenzar por caracterizar de que duelo se está hablando. ¿Cuáles de las innumeras pérdidas cotidianas tienen ese estatuto? Perder un trabajo al que dedicamos veinte años cuando tenemos más de cincuenta, quizás pueda provocar una situación de duelo que exija más tiempo del preconizado por el DSM-IV, inclusive porque cada nuevo fracaso representa una nueva situación de duelo. Los procesos inmigratorios implican duelo, los amigos perdidos implican duelo, la muerte de un cónyuge o un hijo implican duelo. Aunque cada situación es diferente, para el DSM-IV el duelo "normal" es aquel que puede ser elaborado en un período de dos meses.

La desconsideración de la necesaria y siempre particular interrelación individuo-medio, considerar a los sujetos como normales o patológicos independientemente de las frustraciones, infidelidades y riesgos que el médio impone a todo ser vivo (desde la ameba al hombre) conduce inevitablemente a inconsistencias y debilidades epistemológicas.

Recordemos que una de las premisas básicas establecidas por Canguilhem en "Lo Normal y lo patológico" es la siguiente: "El ser vivo y el medio no pueden ser llamados de normales si son considerados separadamente" (Canguilhem, 1993, p.147); sólo se puede afirmar que un ser vivo es normal si lo vinculamos a su medio, si consideramos las soluciones morfológicas, funcionales, sociales y vitales, a partir de las cuáles responde a las demandas que su medio le impone. "Lo normal es poder vivir en un medio en el que fluctuaciones, infidelidades y nuevos acontecimientos son posibles" (Canguilhem, 1993, p.146).

Los criterios de diagnóstico para la depresión establecidos por el DSM-IV, se refieren a conductas genéricas de sujetos indefinidos: viejos o jóvenes, pobres o ricos, desempleados o empresarios. Esto es, las conductas son evaluadas ignorando las demandas que, en cada caso concreto, el medio impone a cada sujeto.

Las múltiples respuestas posibles que podemos dar a los sufrimientos y frustraciones de nuestra vida, eso que para toda la tradición ética occidental nos define como sujetos morales, fueron reducidas por el DSM-IV a comportamientos normales o patológicos. Si respondemos a nuestros sufrimientos, sea aislamiento social; desempleo; falta de reconocimiento profesional; asedio moral en el trabajo; transporte público precario o inexistente; abandono; etc., sin alteraciones de humor, sin sentimiento de frustración, sin alteraciones de apetito o sueño entonces ingresaremos al selecto círculo de los "normales". Si por el contrario, demostramos fatiga y desgano, sentimientos de irritación y frustración, alteraciones en el sueño o el apetito (por un período mayor de dos semanas) en situaciones semejantes, seremos diagnosticados como depresivos.

En relación al par norma-frecuencia, el diagnóstico de depresión presenta una segunda debilidad epistemológica.

Cuando nos referimos a funciones orgánicas, la frecuencia deseable surge como resultado de estudios bio-estadísticos referidos a medias poblacionales. Así, los valores considerados normales para el ritmo cardíaco de 60-80 por minuto, representan la media poblacional. Canguilhem señaló la dificultad de identificar ese valor con lo "normal" y los desvíos con procesos patológicos. Se refiere a Napoleón (Canguilhem, 1993) cuyo ritmo cardíaco, de cuarenta pulsaciones por minuto, no le impidió responder a las demandas de un emperador.

Con la depresión ocurre algo inverso, cuando nos referimos a frecuencia media poblacional todos somos Napoleón, esto es, la media poblacional es más próxima al desvío o anomalía que a la "Norma". Todos tenemos oscilaciones de sueño y apetito, todos tenemos sentimientos de frustración que pueden ser más o menos constantes en determinados períodos de nuestra vida, todos tenemos sentimientos de culpa y todos sentimos sentimientos de fracaso. Esto implica que todos (como Napoleón) representamos un desvío con relación a la media poblacional.

Ocurre entonces una inversión del esquema explicativo clásico. Cuando hablamos de presión arterial o de nivel de colesterol es la frecuencia lo que determina lo deseable. 
En el caso de la depresión ese esquema se invierte, son los comportamientos socialmente deseables (no tener oscilaciones de sueño o apetito, ni sufrimientos, frustraciones o culpa) lo que determina lo normal y es a partir de ese "deber ser" que la frecuencia de conductas se establece como normal o patológica. La fragilidad para determinar los valores biológicos normales a partir de estudios estadísticos analizada por Canguilhem, fue substituida, en el caso de la depresión, por una fragilidad aún mayor. Ya no se trata de definir la frecuencia deseable a partir de la media poblacional. En el caso de la depresión el número deseable de conductas y sentimientos indeseables (cinco sobre nueve en dos semanas) resulta de aquello que los psiquiatras de la American Psiquiatric Asociation definieron por consenso como "normal". Ese consenso define cuando la frecuencia de aparición de un sentimiento o comportamiento es normal y cuando es patológica ( Amaral, 2004; Pignarre, 2001).

El diagnóstico de depresión presenta una tercera fragilidad epistemológica vinculada con la asociación norma-frecuencia.

Como afirma Pignarre (2001) esta es una de las pocas enfermedades donde riesgo y enfermedad se identifican. Las mismas condiciones exigidas para hablar de riesgo de contraer la depresión son las que definen a la depresión leve. Sabemos que no es posible identificar "ser un fumador" y "estar enfermo de cáncer de pulmón", o "comer grasa" y "tener una enfermedad coronaria", en el caso de la depresión un sentimiento de frustración o culpa es al mismo tiempo condición de posibilidad, indicador de riesgo y un síntoma que permite establecer el diagnóstico de la depresión leve.

Señalamos aquí, tres problemas epistemológicos del diagnóstico de depresión propuesto por el DSM-IV que podemos resumir como: (a) reducir la polaridad individuo-medio a conductas y sentimientos de sujetos sin entorno; (b) definir la frecuencia de comportamientos que deben ser considerados normales o patológicos por consenso a partir de lo que se considera socialmente deseable; (c) identificar conductas de riesgo y diagnóstico.

Pero, el diagnóstico de depresión no pretende ser exclusivamente descriptivo o comportamental. Se dirá que es posible establecer explicaciones causales o etiológicas de esta patología por referencia a disfunciones cerebrales, específicamente por referencia al déficit de ciertos neurotransmisores.

\section{Explicación etiológica y localización cerebral}

Los criterios descriptivos de diagnóstico del DSM-IV no agotan el fenómeno patológico de la depresión. Se repite, una y otra vez, que lo que limita todo y cualquier "capricho diagnóstico" (Kitcher, 2002), no son los criterios descriptivos sino las explicaciones etiológicas, la referencia a estudios biológicos de laboratorio que indican que el déficit de ciertos neurotrasmisores, como la serotonina, es la causa directa de la enfermedad.

Las explicaciones causales de la depresión se inscriben en un espacio teórico que posee una lógica completamente diferente de la que caracteriza a los criterios descriptivos de conductas. Ya no se trata de diferenciar lo normal y lo patológico por la frecuencia de aparición de comportamientos indeseados, ahora se trata de realizar, finalmente, el sueño de Charcot (Foucault, 2003). Esto es, localizar en el cuerpo, específicamente en el cerebro, la causa de los síntomas.

La lógica que se pretende aplicar no es otra que aquella que dio nacimiento a la medicina moderna: la localización anatomopatológica de déficit o lesiones en el cuerpo. Sin embargo, este esquema explicativo posee, como los criterios de diagnóstico del DSM-IV, serias debilidades epistemológicas que precisan ser cuidadosamente analizadas.

Para que esta clasificación diagnóstica pueda legitimarse epistemológicamente no es suficiente con establecer frecuencias, crear normas y correlaciones. Resulta necesario dar un paso más en dirección a la identificación de causas biológicas y a la creación de estrategias terapéuticas (Hacking, 2006b, 2005).

Los estudios de localización cerebral, sean realizados a partir de esas nuevas "ventanas al cerebro" que son los PET-scanner (Ortega, 2006, p.90), o con el auxilio de modelos animales, descansan sobre la certeza de que existen explicaciones biológicas causales para la depresión semejantes a aquellas que nos permiten identificar la etiología de otras enfermedades. Así como existe una relación causal entre el Tripanosoma Cruzi y la enfermedad de Chagas, las células cancerígenas y el cáncer de pulmón, del 
mismo modo existiría una relación causal entre el déficit de ciertos neurotransmisores (serotonina o noradrenalina) y la aparición de un Episodio Depresivo o de un Trastorno Depresivo Mayor.

Sin embargo, existen diferencias significativas entre estas estructuras explicativas. La explicación etiológica que se construye para una enfermedad infecciosa no tiene el mismo estatuto epistemológico que aquella construida para la depresión. En el caso de una infección se identifica un conjunto de síntomas que pueden ser indicativos de la enfermedad y se realizan estudios de laboratorio para detectar la existencia de determinada bacteria o parásito².

Los estudios microbiológicos y anatomopatológicos permiten inferir, a partir de la identificación de redes causales más o menos complejas, cuál será la terapéutica más eficaz para esa patología. Los microorganismos serán aislados, cultivados e inoculados en animales reproduciendo la enfermedad, y sobre ellos podrán ser realizados estudios que permitan determinar la eficacia de determinado antibiótico.

En el caso de la depresión esta estructura explicativa se encuentra invertida. En los estudios etiológicos clásicos el diagnóstico y la terapéutica tienen un punto en común, lo que Pignarre (1995, p.75) denomina "marcador biológico". Este testigo confiable está ausente en las enfermedades siquiátricas en general y en la depresión en particular, por esa razón, es necesario crear estrategias explicativas diferentes de aquellas que caracterizan a los estudios etiológicos clásicos.

En el caso de la depresión, ocurre una inversión completa de esas explicaciones. Lo que permite articular la trama explicativa no es el diagnóstico o la explicación etiológica sino la terapéutica. Es a partir del antidepresivo que se inicia la búsqueda de causas biológicas. Se argumentará (aunque no existan estudios conclusivos) que es posible, recurriendo a modelos animales o a imágenes cerebrales, identificar cuales son los mecanismos biológicos, los receptores neuronales afectados, y que, de ese modo, se podrá conocer la causa orgánica, cerebral, de los padecimientos (Pignarre, 2006).

Sin embargo, sabemos que la mayor parte de los diagnósticos de depresión es dada por médicos generales a partir de los criterios establecidos por el DSM-IV o CID-10 (Amaral, 2004; Valentini, 2004). El diagnósticos por imagen o los estudios neurológicos pueden ser realizados en un segundo momento. Pero las alteraciones en el sistema nervioso central solo son identificadas, a posteriori, a partir de las modificaciones que los antidepresivos provocan en las conductas.

Es a partir de la mediación del antidepresivo que se puede reconstruir una red causal explicativa. Ante la ausencia de un "marcador biológico", un microorganismo, un parásito, un tejido celular, la red causal se reconstruye a partir de la terapéutica.

Pignarre encuentra un antecedente de esta inversión explicativa en lo que llama de 'hipótesis dopaminérgica de la esquizofrenia'. En ese caso "se asocia el déficit de un neurotransmisor en el cerebro, la dopamina, a la esquizofrenia con el argumento de que los neurolépticos actúan sobre ese neurotransmisor" (Pignarre, 2001, p.115). Lo mismo ocurre con los inhibidores selectivos de re-captación de serotonina, en este caso se asocia el déficit de un neurotransmisor, la serotonina, con la depresión, con el argumento de que los ISRS actúan sobre ese neurotransmisor.

Este tipo de explicación circular es posible porque, a diferencia de las enfermedades infecciosas, no existen informaciones objetivas que permitan operar una transposición del mundo de los sufrimientos humanos al mundo controlado del laboratorio, no existe "marcador biológico", no existe un "testigo confiable" (Pignarre, 1995).

\author{
2 Podrá objetarse que \\ esta red causal simple no \\ puede ser aplicada a \\ todas las enfermedades, \\ que podemos tener en \\ nuestro organismo el \\ bacilo de Koch sin estar \\ enfermos de \\ tuberculosis. Aunque \\ existan personas \\ saludables que albergan \\ en su organismo el bacilo \\ no es posible, al \\ contrario, imaginar un \\ enfermo de tuberculosis \\ sin restos de bacilos en \\ sangre. Como sabemos, \\ el bacilo es una \\ condición necesaria \\ aunque no suficiente \\ para que exista \\ tuberculosis.
}


Los psicotrópicos permiten crear la ilusión de que las patologías mentales en general, y los sufrimientos psíquicos en particular, ingresaron en la lógica localizacionista de los estudios anatomopatológicos.

Las alteraciones biológicas pueden inferirse a partir de la observación de los efectos de los antidepresivos. Estas alteraciones pueden ser estudiadas en laboratorio a partir de modelos animales, posibilitando un conocimiento directo de los efectos que determinada molécula produce en el comportamiento animal. Pueden también ser estudiadas, con el auxilio del Pet Scan, las alteraciones producidas en el cerebro de sujetos que están bajo el efecto de antidepresivos (Ortega, 2006). Todos estos estudios tienen algo en común: los psicotrópicos preceden siempre la búsqueda del origen biológico de la enfermedad.

Si bien estos estudios se mostraron eficientes para crear nuevos medicamentos, todas las hipótesis etiológicas que fueron construidas a partir de esos protocolos de investigación se han mostrado, hasta hoy, poco conclusivas. Aún los defensores más radicales del origen biológico de los disturbios de humor coinciden en afirmar que las causas precisas permanecen indefinidas y que los estudios existentes no son concluyentes (Romeiro, 2003).

Sin embargo, aunque existe cierto consenso sobre la insuficiencia de informaciones confiables de localización cerebral de la depresión, los estudios dedicados a descubrir y perfeccionar nuevos antidepresivos no han dejado de multiplicarse. Si esto es posible es porque uno y otro se mueven en direcciones independientes. La inversión millonaria en investigación de nuevos antidepresivos tiene dos objetivos claros: encontrar medicamentos con menos efectos colaterales que aquel que está en uso y producir nuevos medicamentos para nuevos diagnósticos que surgen por asociación o aproximación entre disturbios (depresión con ansiedad, anorexia nerviosa, depresión con hiperactividad, etc).

A partir de allí se generó una fructífera y millonaria línea de estudio, de financiamiento y de investigación con características propias. Aunque estos estudios se definan como pertenecientes al campo de las investigaciones biológicas clásicas, afirmando que sus protocolos son idénticos a los estudios de cualquier otra enfermedad, para Pignarre no sería correcto hablar de biología. Crea el concepto de "biología menor" (petite biologie) para designar estos conocimientos que permiten producir nuevos psicofármacos a partir de las reacciones provocadas por los antidepresivos existentes.

Como afirma ese autor: "Los investigadores de la industria farmacéutica imponen su biología menor. Ella no tiene gran utilidad fuera del laboratorio, su ambición es perfeccionar y afinar los instrumentos de selección de nuevos psicotrópicos que son siempre los penúltimos" (Pignarre, 2001, p.120). El último descubrimiento siempre viene a remediar los efectos colaterales del anterior y a anunciar los medicamentos por venir, que reducirán los efectos colaterales que este presenta.

Una característica de esa "biología menor" es la identificación diagnóstico-terapéutica, pues es el medicamento el que cumple el papel de "marcador biológico". Así: "pasando de una clase química de antidepresivo a otra, los investigadores y los médicos han modificado los criterios de clasificación de los pacientes, su acción diferenciada permite distinguir: depresivos que tienen necesidad de ser estimulados y aquellos que precisan ser tranquilizados, depresivos ansiosos y depresivos agresivos o totalmente inhibidos" (Pignarre, 2001, p.121).

Las pequeñas alteraciones en las diversas moléculas que son testadas en laboratorio, transforman los criterios de clasificación de pacientes y como consecuencia terminan por transformar la propia definición del diagnóstico de depresión. La falta de un marcador biológico al mismo tiempo que representa el límite y la dificultad de este modelo explicativo, abre ilimitadas posibilidades de estudios futuros. Permite que sea explorada libremente, sin necesidad de marcadores, esa amplia frontera, poco clara e indefinida, entre el sufrimiento normal y el sufrimiento claramente patológico. Permite "explorar el territorio de los comportamientos y las emociones que aún no fueron medicalizados, y de participar de la redefinición permanente de los problemas mentales" (Pignarre, 2001, p.143).

Nos situamos así en un territorio sin límites epistemológicos definidos, sin marcadores biológicos, ni testigos confiables, pero es justamente esa ambigüedad la que posibilita la creciente multiplicación de diagnósticos y, consecuentemente, la creación de la misteriosa y temida epidemia de depresión. 


\section{A modo de conclusión}

Para poder realizar un análisis epistemológico de la categoría diagnóstica de depresión fue necesario diferenciar dos niveles discursivos.

Un nivel descriptivo que corresponde a la definición de los criterios de identificación de la enfermedad establecidos por el DSM-IV. Señalamos algunos problemas epistemológicos que caracterizan a ese diagnóstico. Fundamentalmente, que los criterios establecidos por el DSM-IV, para ser cuantificados precisan referirse a sujetos sin entorno. En ese marco, no es posible considerar que cada uno de nuestros sufrimientos es una respuesta a una infidelidad, frustración o dificultad que nuestro medio nos impone.

Los criterios definidos por el DSM-IV no son suficientes para caracterizar el diagnóstico. Es necesario introducir un segundo nivel discursivo, ya no descriptivo sino etiológico. Para ello será necesario localizar en el cuerpo (Hacking, 2006b), en los neurotransmisores, la etiología de la enfermedad. Establecer relaciones causales propias de lo que Pignarre denominó una "biología menor", esa red explicativa sólo puede ser reconstruida con la mediación de los antidepresivos.

La explicación etiológica "a posteriori" de la depresión se asemeja a la tentativa de identificación de los mecanismos neurológicos afectados por ciertas drogas que alteran comportamientos. Sabemos que frente una situación de inhibición en un evento social, el consumo moderado de bebida alcohólica puede transformarnos en sujetos desinhibidos por acción del alcohol en el sistema nervioso central. Sin embargo, esa certeza no nos autoriza ni a patologizar la inhibición, ni a considerar el consumo de bebidas alcohólicas como terapéutico, ni a considerar que la inhibición es el resultado del déficit de alcohol en el sistema nervioso. Por el contrario, una reacción favorable a un ISRS, permite afirmar que un sentimiento de tristeza es patológico, que la terapéutica apropiada es el ISRS y, en consecuencia, que la depresión es el resultado del déficit de serotonina.

Es a partir de la mediación del psicofármaco que los dos niveles discursivos, descriptivo y etiológico, se articulan. El antidepresivo tiene un efecto doble: permite definir la etiología (ISRS-déficit de serotonina) y permite optimizar y crear nuevos criterios diagnósticos.

Sabemos que existen clasificaciones que, una vez integradas a la vida de los individuos, modifican el modo como ellos se observan a sí mismos. Las clasificaciones humanas generan efectos en los sujetos, cada clasificación, cada diagnóstico, cada tipificación de personas, implica un cambio en el modo como actuamos, como expresamos nuestras emociones y sentimientos, en el modo, en fin, de construirnos como sujetos.

Nuestra sociedad parece haber definido un modo privilegiado y socialmente aceptado de sufrir, un modo medicalizado de administrar nuestros fracasos y angustias. Como consecuencia, se admite de forma pasiva y acrítica un diagnóstico que, aún padeciendo de serias limitaciones epistemológicas, nos permite crear la ilusión de que vivir es indoloro (Valerim, 2007).

Así, y a pesar de las limitaciones epistemológicas aquí señaladas, el diagnóstico de depresión resulta extremamente eficaz para alcanzar el objetivo de "clasificar" y "modelar personas" (Hacking, 2000). Esa clasificación lleva a patologizar nuestros sufrimientos, tal como ocurre con varios diagnósticos de depresión leve o moderada, modelando subjetividades con serias incapacidades para aprender de sus fracasos y angustias. Al mismo tiempo, nos conduce a privilegiar un modo de sufrir donde la palabra y el diálogo, aquello que define la condición humana, está subordinado al uso de medicamentos, produciendo sujetos que deben administrar sus sufrimientos de modo silencioso y solitario.

\author{
${ }^{3}$ Para que este primer \\ nivel de identificación y \\ descripción pueda \\ consolidarse, el discurso \\ médico y psiquiátrico \\ debió valerse de los \\ cuatro primeros motores \\ de descubrimiento \\ enunciados por Hacking. \\ Fue necesario, contary \\ establecer correlaciones \\ que evidencien el \\ acelerado crecimiento \\ de la enfermedad y su \\ vinculación con \\ diferentes etapas, \\ circunstancias y hábitos \\ de nuestras vidas. Se \\ debió cuantificar y crear \\ normas con la finalidad \\ de establecer padrones \\ deseables de frecuencia \\ de aparición de \\ conductas y desvíos \\ patológicos que \\ permitan identificar la \\ enfermedad.
}




\section{Referencias}

AMARAL, A. A psiquiatria no divão. Rio de Janeiro: Relume Dumará, 2004.

AMERICAN PSYCHIATRIC ASSOCIATION. APA. Manual de diagnóstico e estatística das perturbações mentais. 4.ed. Washington: APA, 1994.

BOORSE, C. On the distinction between disease and illnes. Philos. Public Affairs, n.5, p.40-68, 1975.

BRASIL. Ministério da Saúde. Portal da Saúde. Disponível em: <http://

portal.saude.gov.br/portal/saude/area.cfm?id_area=124>. Acesso em: 28 abr. 2007.

CANGUILHEM, G. Le normal et le pathologique. Paris: PUF, 1993.

La santé. Paris: Sables, 1990.

FOUCAULT, M. Le pouvoir psyquiatrique. Paris: Gallimard, 2003.

FRANCIA, M.D.S. Info-deppression: en savoir plus pour en sortir. Paris: Gov. de Francia, 2007.

FRANCO, G.P. Qualidade de vida e sintomas depressivos em residentes de enfermagem. Rev. Latino-am. Enferm., v.13, n.2, p.139-44, 2005.

GOFFMAN, E. Estigma: notas sobre a manipulação da identidade deteriorada. Rio de Janeiro: LTC, 1988.

HACKING, I. Kinds of people: moving targets. London: The British Academy, 2006a.

L'âme réécrite: étude sur la personnalité multiple et les sciences de la mémoire. Paris: Les Empêcheurs de penser en rond, 2006b.

Philosophie et Histoire des conceptes scientifiques. In: gens. Paris: College de France, 2005. p.593-613.

Façonner les

Leçon inaugurale du College de France. Paris: Collège de France, 2001.

Façonner les gens: le seuil de pauvreté. In: BEAUD, J.P.; PRÉVOST, J.G. (Eds.).

L'ère du chiffre: systèmes statistiques et traditions nationales. Québec: Presses de I'Université de Québec, 2000. p.17-36.

KITCHER, P. Las vidas por venir. México: Universidad Nacional Autonoma de México, 2002.

LECOURT, D. Normas. In: RUSSO, M.; CAPONI, S. (Eds.). Estudos de filosofia e história das ciências biomédicas. São Paulo: Discurso, 2006. p.290-325.

LOBO, I. Depressão é parceira indesejável de $10 \%$ dos idosos. Brasília: Ministério da Saúde, 2007.

NIETZSCHE, F. La gaya Ciencia. México: Ed. Mexicanos Unidos, 1981.

NORDENFELT, L. On the circle of health. In: ; LINDAHL, I. (Eds.). Health, disease, and causal explanations in medicine. Boston: D. Reidel Publishing Company, 1984. p.60. [Philosophy and Medicine]

ORTEGA, F. O corpo transparente: visualização médica e cultura popular no século XX. Hist., Cienc., Saude - Manguinhos, v.13, supl., p.89-107, 2006.

PIGNARRE, P. Les malheurs des psys: psychotropes et médicalisation du social. Paris: La Découverte, 2006.

Comment la dépression est devenue une épidémie. Paris: Hachette, 2001.

Les deux médecines: médicaments, psychotropes et suggestion thérapeutique. Paris: La Découverte, 1995. 
ROMEIRO, L.A.S. Novas estratégias terapéuticas para o tratamento da depressão: uma visão da química médica. Quim. Nova, v.26, n.3, p.352-8, 2003.

VALENTINI, W.L. et al. Treinamento de clínicos para o diagnóstico e tratamento da depressão. Rev. Saude Publica, v.38, n.4, p.524-8, 2004.

VALERIM, S. Deixar a ilusão de que viver é indolor. Mil e uma forma de ser objeto. In: JORNADA DE SEÇÃO DE PSICANÁLISE DE SANTA CATARINA, 2., 2007, Cidade. Anais... Cidade: Escola Brasileira de Psicanálise, 2007.

WHO. WORLD HEALTH ORGANIZATION. Mental health: depression. Geneva: WHO, 2007.

CAPONI, S. Uma análise epistemológica do diagnóstico de depressão. Interface Comunic., Saúde, Educ., v.13, n.29, p.327-38, abr./jun. 2009.

Tomando como ponto de partida as reflexões de autores como Canguilhem, Hacking e Pignarre, discute-se o diagnóstico de depressão. Não se pretende negar a existência de situações concretas que possam corresponder a esse diagnóstico, mas sim realizar uma análise estritamente epistemológica, e necessariamente incompleta, do modo como os diferentes aspectos desta classificação se validam e legitimam. Em primeiro lugar, se analisam, com base nas teorizações de Hacking, de que modo se constroem as classificações humanas. Em segundo lugar, com base nos conceitos de normalidade e patologia de Canguilhem, destacam-se algumas fragilidades epistemológicas do diagnóstico descritivo de depressão presentes no DSM-IV. Por fim, tomando por base os estudos de Pignarre, são analisadas algumas dificuldades próprias das explicações etiológicas dadas a essa patologia. É possível concluir que o alarmante crescimento desse diagnóstico está associado à fragilidade epistemológica dessa categoria classificatória.

Palavras-chave: Depressão. Epistemologia. Classificações diagnósticas. Causalidade.

Recebido em 02/07/08. Aprovado em 25/10/08. 